\title{
PSEUDOSPECTRAL OPERATORS AND THE POINTWISE ERGODIC THEOREM
}

\author{
R. E. BRADLEY
}

(Communicated by Palle E. T. Jorgensen)

\begin{abstract}
We show that for a class of operators which properly contains the normal operators on $L_{2}$,

$$
\frac{1}{n} \sum_{i=0}^{n-1} T^{i} f \rightarrow \text { a.e. iff } \frac{1}{2^{n}} \sum_{i=0}^{2^{n}-1} T^{i} f \rightarrow \text { a.e. }
$$

This theorem is used to give an alternate form of a theorem of Gaposhkin concerning the pointwise ergodic theorem for normal operators.
\end{abstract}

\section{INTRODUCTION}

In [G3], V. F. Gaposhkin gives necessary and sufficient conditions for the pointwise ergodic theorem for normal contractions. This remarkable theorem, which is local in the sense that it is specific to a given pair of operator $T$ and $L_{2}$ function $f$, uses spectral theory.

Perhaps as remarkable as the theorem itself is the result that

$$
\frac{1}{n} \sum_{i=0}^{n-1} T^{i} f
$$

converges a.e. if and only if

$$
\frac{1}{2^{n}} \sum_{i=0}^{2^{n}-1} T^{i} f
$$

converges a.e. Although this is not explicitly stated, the proof is contained in the details of [G1], when interpreted in the light of [G3]. In [G2], Gaposhkin does remark that the corresponding result holds for Cesàro averages of stationary processes. The analogous result for orthogonal series appears to date back to Tandori $[\mathrm{A}]$.

In this paper, we give a direct proof of this equiconvergence result. We consider the class of pseudospectral operators, which is a proper generalization

Received by the editors January 26, 1990 and, in revised form, April 30, 1990.

1980 Mathematics Subject Classification (1985 Revision). Primary 28D99, 47A35.

Key words and phrases. Spectral measure, normal operators, pointwise ergodic theorem. 
of the class of normal operators. Along the way, we capture the analogous version of Gaposhkin's theorem on equivalent conditions for the pointwise ergodic theorem. It is hoped that this version of Gaposhkin's theorem makes the connection between the pointwise ergodic theorem and convergence of orthogonal series even more transparent.

The author is grateful to M. A. Akcoglu for suggesting this problem and identifying the conditions used in the definition of pseudospectral.

\section{PSEUdospectral operators}

Definition. Suppose $T$ is a linear operator on a Hilbert space $\mathscr{H}$, and $\left\{\alpha_{n}\right\}_{n=0}^{\infty}$ and $\left\{\beta_{n}\right\}_{n=0}^{\infty}$ are two sequences of positive real numbers converging to zero. We say that $T$ is pseudospectral if $\mathscr{H}$ admits an orthogonal decomposition

$$
\mathscr{H}=\overline{\mathscr{H}} \oplus\left(\bigoplus_{n=0}^{\infty} \mathscr{H}_{n}\right),
$$

where $\overline{\mathscr{H}}=\{f \mid T f=f\}$ is the space of $T$-invariant functions and, for each $n \geq 0, \mathscr{H}_{n}$ is $T$-invariant, with

$$
\alpha_{n}\|f\| \leq\|T f-f\| \leq \beta_{n}\|f\|
$$

for every $f \in \mathscr{H}_{n}$.

Proposition. For any strictly decreasing sequence $\left\{\gamma_{n}\right\}_{n=0}^{\infty}$ converging to zero and any bounded normal operator $T, T$ is pseudospectral with $\alpha_{n}=\beta_{n+1}=\gamma_{n}$ for $n \geq 0$.

Proof. We use the spectral theorem in the following form: there is a finite measure space $(X, \mathscr{F}, \mu)$ and a function $\phi \in L_{\infty}(X, \mathscr{F}, \mu)$ such that the action of $T$ on $\mathscr{H}$ is unitarily equivalent to multiplication by $\phi$ on $L_{2}(X, \mathscr{F}, \mu)$; see $[\mathrm{H}]$. Explicitly, there is a unitary operator $U: \mathscr{H} \rightarrow L_{2}$ such that for every $f \in L_{2},\left(U T U^{-1} f\right)(x)=\phi(x) f(x)$.

Let

$$
\begin{gathered}
\bar{A}=\{x \in X \mid \phi(x)=1\}, \\
A_{0}=\left\{x \in X|| \phi(x)-1 \mid>\gamma_{0}\right\}
\end{gathered}
$$

and, for each $n \geq 1$,

$$
A_{n}=\left\{x \in X\left|\gamma_{n}<\right| \phi(x)-1 \mid \leq \gamma_{n-1}\right\} .
$$

Let $\overline{\mathscr{G}}=L_{2}(\bar{A})$ and $\mathscr{G}_{n}=L_{2}\left(A_{n}\right)$ for $n \geq 0$, where, for $E \in \mathscr{F}, L_{2}(E)$ is the $L_{2}$ space of the restricted measure space $\left(E, \mathscr{F}_{E}, \mu_{E}\right)$, with both the measure and the $\sigma$-algebra of measurable sets suitably restricted. It is clear that $U T U^{-1}$ is a pseudospectral operator on $L_{2}$ with respect to the above orthogonal decomposition and the sequences $\left\{\alpha_{n}\right\}_{n=0}^{\infty}$ and $\left\{\beta_{n}\right\}_{n=0}^{\infty}$ as defined in statement of the theorem (we may take $\beta_{0}=\|T\|+1$ ). This pulls back to $T$ and $\mathscr{H}$ via $U^{-1}$, completing the proof.

Remark. Theorems 1 and 2, which follow, will therefore hold for any normal operator with $\gamma_{n}=2^{-n}$. 
Proposition. There exist nonnormal pseudospectral operators.

Proof. Suppose a normal operator $T$ on a Hilbert space $\mathscr{H}$ and a sequence $\left\{\gamma_{n}\right\}_{n=0}^{\infty}$ are given. Let $\overline{\mathscr{H}}$ and $\mathscr{H}_{n}$ be the decomposition corresponding to $\left\{\gamma_{n}\right\}_{n=0}^{\infty}$, as in the proof above. Let $\mathscr{G}_{0}$ be a two-dimensional Hilbert space and let $S_{0}$ be multiplication by

$$
\left[\begin{array}{ll}
1 & 1 \\
4 & 1
\end{array}\right]
$$

Define $\mathscr{G}=\mathscr{G}_{0} \oplus \mathscr{H}$ and $S=S_{0} \oplus T$. Then one verifies that $S$ is pseudospectral with the decomposition $\overline{\mathscr{G}}=\overline{\mathscr{H}}, \mathscr{G}_{n+1}=\mathscr{H}_{n}$, for $n \geq 0$ and with $\alpha_{n+1}=$ $\beta_{n+2}=\gamma_{n}$ for $n \geq 0, \beta_{1}=\|T\|+1, \alpha_{0}=1$, and $\beta_{0}=4$. However, $S$ is not normal, since $S_{0}$ is not.

\section{SOME UPPER BOUNDS}

Definition. A linear operator $T$ is called a contraction if $\|T\| \leq 1$; equivalently, if $\|T f\| \leq\|f\|$ for every $f \in \mathscr{H}$. Given a linear operator $T$ on $\mathscr{H}$ and $f \in \mathscr{H}$,

$$
A_{n} f=\frac{1}{n} \sum_{i=0}^{n-1} T^{i} f
$$

Lemma. If $T$ is a contraction of a Hilbert space $\mathscr{H}$ and $f \in \mathscr{H}$, then for every $n, k \geq 1$,

$$
\left\|A_{n+k} f-A_{n} f\right\| \leq \frac{2 k}{n+k}\|f\|
$$

Proof.

$$
\begin{aligned}
\left\|A_{n+k} f-A_{n} f\right\| & =\left\|\left(\frac{1}{n+k}-\frac{1}{n}\right) \sum_{i=0}^{n-1} T^{i} f-\frac{1}{n+k} \sum_{i=n}^{n+k-1} T^{i} f\right\| \\
& \leq \frac{k}{n(n+k)} \sum_{i=0}^{n-1}\left\|T^{i} f\right\|+\frac{1}{n+k} \sum_{i=n}^{n+k-1}\left\|T^{i} f\right\| \\
& \leq \frac{k}{n+k}\|f\|+\frac{k}{n+k}\|f\|,
\end{aligned}
$$

where we have used the fact that $\left\|T^{i} f\right\| \leq\|f\|$ for all powers of a contraction.

Lemma. Suppose $T$ is a contraction of a Hilbert space $\mathscr{H}$ and that $\|T f-f\| \geq$ $\alpha\|f\|$ for every $f \in \mathscr{H}$. If $n, k \geq 1$, then

$$
\begin{gathered}
\left\|A_{n} f\right\| \leq \frac{2\|f\|}{n \alpha}, \\
\left\|A_{n+k}-A_{n} f\right\| \leq \frac{4\|f\|}{n \alpha} .
\end{gathered}
$$


Proof.

Therefore,

$$
\frac{1}{n}\left\|T^{n} f-f\right\|=\left\|T\left(A_{n} f\right)-A_{n} f\right\| \geq \alpha\left\|A_{n} f\right\|
$$

$$
\left\|A_{n} f\right\| \leq \frac{\left\|T^{n} f-f\right\|}{n \alpha} \leq \frac{2\|f\|}{n \alpha} .
$$

The bound (B3) follows from (B2) and the triangle inequality.

Definition. Given $n, k \geq 1$, let $C_{n, k}$ be the $n \times(n+k)$ matrix with entries $c_{i, j}$ for $0 \leq i \leq n-1$ and $0 \leq j \leq n+k-1$, satisfying

$$
c_{0, j}= \begin{cases}1 & \text { if } j=0 \\ \min \left\{1, \frac{n+k}{n}-\sum_{m=0}^{j-1} c_{0, m}\right\} & \text { otherwise }\end{cases}
$$

and, for $1 \leq i \leq n-1$,

$$
c_{i, j}= \begin{cases}1-\sum_{m=0}^{i-1} c_{m, j} & \text { if } \sum_{m=0}^{i-1} c_{m, j}>0, \\ \min \left\{1, \frac{n+k}{n}-\sum_{m=0}^{j-1} c_{i, m}\right\} & \text { otherwise }\end{cases}
$$

Example.

$$
C_{5,3}=\left[\begin{array}{cccccccc}
1 & 0.6 & 0 & 0 & 0 & 0 & 0 & 0 \\
0 & 0.4 & 1 & 0.2 & 0 & 0 & 0 & 0 \\
0 & 0 & 0 & 0.8 & 0.8 & 0 & 0 & 0 \\
0 & 0 & 0 & 0 & 0.2 & 1 & 0.4 & 0 \\
0 & 0 & 0 & 0 & 0 & 0 & 0.6 & 1
\end{array}\right]
$$

Definition. $\lfloor x\rfloor$ is the largest integer less than or equal to $x .\lceil x\rceil$ is the smallest integer greater than or equal to $x$. The following lemma is immediate:

Lemma. If $n, k \geq 1$ then
(a) $\sum_{i=0}^{n-1} c_{i, j}=1$,
whenever $0 \leq j \leq n+k-1$,
(b) $\sum_{j=0}^{n+k-1} c_{i, j}=\frac{n+k}{n}$
whenever $0 \leq i \leq n-1$,
(c) $c_{i, j}=0$
unless $\left\lfloor\frac{i(n+k)}{n}\right\rfloor \leq j \leq\left\lceil\frac{(i+1)(n+k)}{n}-1\right\rceil$.

Lemma. Suppose $T$ is a contraction of a Hilbert space $\mathscr{H}$ and that $\|T f-f\| \leq$ $\beta\|f\|$ for every $f \in \mathscr{H}$. If $n, k \geq 1$, then

$$
\begin{gathered}
\left\|A_{n} f-f\right\| \leq n \beta\|f\|, \\
\left\|A_{n+k} f-A_{n} f\right\| \leq 2 k \beta\|f\| .
\end{gathered}
$$


Proof. For (B4), observe that

$$
A_{n} f-f=\frac{1}{n} \sum_{i=1}^{n-1} i\left(T^{n-i} f-T^{n-i-1} f\right) .
$$

Therefore,

$$
\left\|A_{n} f-f\right\| \leq \frac{1}{n} \sum_{i=1}^{n-1} i \beta\|f\|=\frac{(n-1) \beta\|f\|}{2} .
$$

For (B5), we make use of the previous lemma. On the one hand,

$$
A_{n+k} f=\frac{1}{n+k} \sum_{j=0}^{n+k-1}\left(\sum_{i=0}^{n-1} c_{i, j}\right) T^{j} f=\frac{1}{n+k} \sum_{i=0}^{n-1} \sum_{j=0}^{n+k-1} c_{i, j} T^{j} f
$$

follows from part (a). On the other hand, part (b) implies that

$$
A_{n} f=\frac{1}{n+k} \sum_{i=0}^{n-1} \frac{n+k}{n} T^{i} f=\frac{1}{n+k} \sum_{i=0}^{n-1} \sum_{j=0}^{n+k-1} c_{i, j} T^{i} f .
$$

Now observe that $\left\|T^{i} f-f\right\| \leq i \beta\|f\|$, by a simple induction. Let $l_{i}=$ $\lfloor i(n+k) / n\rfloor$ and $m_{i}=\left\lceil\frac{(i+1)(n+k)}{n}-1\right\rceil$. Then by part (c),

$$
\begin{aligned}
\left\|A_{n+k} f-A_{n} f\right\| & =\frac{1}{n+k}\left\|\sum_{i=0}^{n-1} \sum_{j=l_{i}}^{m_{i}} c_{i, j}\left(T^{j} f-T^{i} f\right)\right\| \\
& \leq \frac{1}{n+k} \sum_{i=0}^{n-1} \sum_{j=l_{i}}^{m_{i}} c_{i, j}\left\|T^{j-i} f-f\right\| \\
& \leq \frac{1}{n+k} \sum_{i=0}^{n-1} \sum_{j=l_{i}}^{m_{i}} c_{i, j}\left(m_{i}-i\right) \beta\|f\| \\
& =\frac{1}{n} \sum_{i=0}^{n-1}\left[\frac{(i+1)(n+k)}{n}-(i+1)\right] \beta\|f\| \\
& \leq \frac{\beta\|f\|}{n} \sum_{i=0}^{n-1}\left(\frac{(i+1) k}{n}+1\right) \\
& =\left(\frac{(n+1) k}{2 n}+1\right) \beta\|f\|,
\end{aligned}
$$

which completes the proof.

\section{THE MAIN THEOREM}

Theorem 1. Suppose $T$ is a pseudospectral contraction on $L_{2}(X, \mathscr{F}, \mu)$ with constants $M$ and $N$ such that $\alpha_{n} 2^{n} \geq M$ and $\beta_{n} 2^{n} \leq N$ for every $n \geq 0$. If $f \in L_{2}(X, \mathscr{F}, \mu)$, then

$\lim _{m \rightarrow \infty} A_{m} f$ exists a.e. if and only if $\lim _{m \rightarrow \infty} A_{2^{m}} f$ exists a.e. 
Proof. The implication is immediate. To prove the converse, it is sufficient to show that

$$
\sum_{m=1}^{\infty}\left\|\phi_{m}\right\|^{2}<\infty
$$

where

$$
\phi_{m}(x)=\max _{1 \leq k<2^{m}}\left|\left(A_{2^{m}+k} f\right)(x)-\left(A_{2^{m}} f\right)(x)\right| .
$$

We desire a bound on $\left|\phi_{m}\right|$ which is independent of $k$. This can be done in a variety of ways using terms of the form $\left|A_{i} f-A_{j} f\right|$. If we wish to show that $\sum\left\|\phi_{m}\right\|^{2}<\infty$, though, we must keep both the number of terms and the size of $|i-j|$ small. We also wish to bound $\left|\varphi_{m}\right|^{2}$ without cross terms; that is, with terms of the type $\left|A_{i} f-A_{j} f\right|^{2}$ only. We do this using Gaposhkin's trick. It involves the binary expansion of an arbitrary integer $i, 2^{m}<i<2^{m+1}$, and an application of Hölder's inequality; see [G1]. To represent an integer in this range, one needs a string of $m+1$ bits with a leading 1 . The process of transforming such an integer into the number $2^{m}$ can be thought of as erasing the nonzero bits, other than the leading 1 , one at a time (in other words, by a series of no more than $m$ successive jumps, each size $2^{m-p}$ for some $p, 1 \leq$ $p \leq m)$.

Let

$$
D_{m, p, j} f=\left|A_{2^{m}+j 2^{m-p}} f-A_{2^{m}+(j-1) 2^{m-p}} f\right|,
$$

for each $m \geq 1,1 \leq p \leq m$ and $1 \leq j \leq 2^{p}-1$. For a given $k, 1 \leq k<2^{m}$, let $\varepsilon_{p}^{(k)}$ be the $p$ th bit of $k$, counting from the left and including leading zeroes. Let $j_{p}^{(k)}$ be the number whose binary expansion is the first $p$ bits of the binary expansion of $k$, also counting from the left and including leading zeroes. Then

$$
A_{2^{m}+k} f-A_{2^{m}} f=\sum_{p=1}^{m} \varepsilon_{p}^{(k)} D_{m, p, j_{p}^{(k)}} f .
$$

Therefore,

$$
\begin{aligned}
\left|A_{2^{m}+k} f-A_{2^{m}} f\right|^{2} & \leq\left|\sum_{p=1}^{m} D_{m, p, j_{p}^{(k)}} f\right|^{2} \\
& \leq\left(\sum_{p=1}^{m} \frac{1}{p^{2}}\right)\left(\sum_{p=1}^{m} p^{2}\left|D_{m, p, j_{p}^{(k)}} f\right|^{2}\right) \\
& \leq\left(\frac{\pi^{2}}{6}\right) \sum_{p=1}^{m} p^{2} \sum_{j=1}^{2^{p}-1}\left|D_{m, p, j} f\right|^{2},
\end{aligned}
$$

where Hölder's inequality is used for the second line. Thus,

$$
\left\|\phi_{m}\right\|^{2}=\left\|\left|\phi_{m}\right|^{2}\right\|_{1} \leq C \sum_{p=1}^{m} p^{2} \sum_{j=1}^{2^{p}-1}\left\|D_{m, p, j} f\right\|^{2} .
$$


Consider the orthogonal decomposition of $L_{2}(X, \mathscr{F}, \mu)$ relative to $T$. Let $\bar{f}$ be the projection of $f$ onto $\overline{\mathscr{H}}$ and, for each $n \geq 0$, let $f_{n}$ be the projection of $f$ onto $\mathscr{H}_{n} ; \bar{f}$ is an invariant function and each subspace is $T$-invariant, so we have

$$
\begin{aligned}
\left\|D_{m, p, j} f\right\|^{2}=\sum_{n=0}^{\infty}\left\|D_{m, p, j} f_{n}\right\|^{2} & \\
= & \left(\sum_{n=m+1}^{\infty}+\sum_{n=m-p+1}^{m}+\sum_{n=0}^{m-p}\right)\left\|D_{m, p, j} f_{n}\right\|^{2} \\
\quad \leq & C^{\prime}\left[\sum_{n=m+1}^{\infty}\left(2^{m-p} \beta_{n}\left\|f_{n}\right\|\right)^{2}+\sum_{n=m-p+1}^{m}\left(2^{-p}\left\|f_{n}\right\|\right)^{2}+\sum_{n=0}^{m-p}\left(2^{-m} \alpha_{n}^{-1}\left\|f_{n}\right\|\right)^{2}\right] \\
& \stackrel{\text { def }}{=} C^{\prime}\left[\Sigma_{1, m, p}+\Sigma_{2, m, p}+\Sigma_{3, m, p}\right],
\end{aligned}
$$

where we have used the upper bounds (B5), (B1), and (B3), respectively, in the third line. Therefore,

$$
\begin{aligned}
\sum_{m=1}^{\infty}\left\|\phi_{m}\right\|^{2} & \leq C^{\prime \prime} \sum_{m=1}^{\infty} \sum_{p=1}^{m} p^{2} 2^{p}\left(\Sigma_{1, m, p}+\Sigma_{2, m, p}+\Sigma_{3, m, p}\right) \\
& \stackrel{\text { def }}{=} \Sigma_{1}+\Sigma_{2}+\Sigma_{3} .
\end{aligned}
$$

The proof is completed by showing that each $\Sigma_{i}$ is finite. This is done by changing the order of summation, using the bounds $\alpha_{n} 2^{n} \geq M$ and $\beta_{n} 2^{n} \leq N$ for every $n \geq 0$ as well as the properties of geometric series. The details may be found in [G1].

Remarks. (1) The theorem holds with the sequence $b^{n}$, where $b$ is an integer greater than 1 , everywhere replacing $2^{n}$. The expansion of $k$ to the base $b$ is used instead of the binary expansion.

(2) One may show that $\sum_{m=1}^{\infty}\left\|\phi_{m}\right\|^{p}<\infty$ for any $p>1$ such that $\sum_{n=0}^{\infty}\left\|f_{n}\right\|^{p}<\infty$; the details are the same.

\section{AN EQUIVALENT OF THE POINTWISE ERGODIC THEOREM}

Definition. If $T$ is a pseudospectral operator with corresponding orthogonal decomposition

$$
\mathscr{H}=\overline{\mathscr{H}} \oplus\left(\bigoplus_{n=0}^{\infty} \mathscr{H}_{n}\right),
$$

let $P_{n}$ denote the projection onto the tail subspace $\bigoplus_{k=n}^{\infty} \mathscr{H}_{k}$.

Theorem. Suppose $T$ is a pseudospectral contraction on $L_{2}(X, \mathscr{F}, \mu)$ with constants $M$ and $N$ such that $\alpha_{n} 2^{n} \geq M$ and $\beta_{n} 2^{n} \leq N$ for every $n \geq 0$. If $f \in L_{2}(X, \mathscr{F}, \mu)$, then

$$
\lim _{m \rightarrow \infty} A_{n} f \text { exists a.e. if and only if } \lim _{m \rightarrow \infty} P_{n} f=0 \text { a.e. }
$$


Proof. Let $f \in L_{2}(X, \mathscr{F}, \mu)$. As in the previous proof, let $\bar{f}$ be the projection of $f$ onto $\overline{\mathscr{H}}$ and, for each $n \geq 0$, let $f_{n}$ be the projection of $f$ onto $\mathscr{H}_{n}$. Let $S_{n}=A_{2^{n}} f-\bar{f}-P_{n+1} f$. We will show that $S_{n} \rightarrow 0$ a.e. This implies that $A_{2^{n}} f \rightarrow \bar{f}$ iff $P_{n} f \rightarrow 0$. Finally, we have $A_{n} f \rightarrow \bar{f}$ iff $P_{n} f \rightarrow 0$ by the previous theorem.

Observe that

$$
S_{n}=\sum_{k=0}^{n} A_{2^{n}} f_{k}+\sum_{k=n+1}^{\infty}\left(A_{2^{n}} f_{k}-f_{k}\right) .
$$

Because of the upper bound (B2), we have

$$
\left\|A_{2^{n}} f_{k}\right\| \leq C_{1} 2^{-n+k}\left\|f_{k}\right\| \text {. }
$$

Becalise of (B4),

Therefore,

$$
\left\|A_{2^{n}} f_{k}-f_{k}\right\| \leq C_{2} 2^{n-k}\left\|f_{k}\right\|
$$

$$
\begin{aligned}
\sum_{n=0}^{\infty}\left\|S_{n}\right\|^{2} & \leq C_{1} \sum_{n=0}^{\infty} \sum_{k=0}^{n}\left(2^{-n+k}\left\|f_{k}\right\|\right)^{2}+C_{2} \sum_{n=0}^{\infty} \sum_{k=n+1}^{\infty}\left(2^{n-k}\left\|f_{k}\right\|\right)^{2} \\
& \leq C_{1} \sum_{k=0}^{\infty} 2^{2 k}\left\|f_{k}\right\|^{2} \sum_{n=k}^{\infty} 2^{-2 n}+C_{2} \sum_{k=1}^{\infty} 2^{-2 k}\left\|f_{k}\right\|^{2} \sum_{n=0}^{k-1} 2^{2 n} \\
& \leq\left(2 C_{1}+C_{2}\right) \sum_{k=0}^{\infty}\left\|f_{k}\right\|^{2}<\infty .
\end{aligned}
$$

Thus $S_{n} \rightarrow 0$ a.e., as desired.

Remark. In [B], Burkholder showed, by adapting an example, due to Menchoff, of a function whose orthogonal series does not converge a.e. (in fact, diverges a.e.), that the pointwise ergodic theorem does not hold for an arbitrary (i.e., nonpositive) contraction of $L_{2}$. By Theorem 2, we see that in some sense this is the only reason a function may fail to satisfy the pointwise ergodic theorem, at least in the case of pseudospectral operators.

\section{REFERENCES}

[A] G. Alexits, Problems in the convergence of orthogonal series, Pergamon Press, New York, 1961.

[B] D. Burkholder, Semi-Gaussian subspaces, Trans. Amer. Math. Soc. 104 (1962), 123-131.

[G1] V. F. Gaposhkin, A theorem on the convergence almost everywhere of a sequence of measurable functions, and its application to sequences of stochastic integrals, Math. USSR Sbornik 33 (1977), 1-19. (English transl.)

[G2] _ Criteria for the strong law of large numbers for some classes of second-order stationary processes and homogeneous random fields, Theory Probab. and Appl. 22 (1977), 286-310. (English transl.)

[G3] _ Individual ergodic theorem for normal operators in $L_{2}$, Functional Anal. Appl. 15 (1981), 14-18. (English transl.)

[H] P. R. Halmos, What does the spectral theorem say? Amer. Math. Monthly 70 (1963), 241-247.

Department of Mathematics, Northwestern University, Evanston, Illinois 60208 